\title{
Characterization of the left ventricular arrhythmogenic substrate with multimodality imaging: role of innervation imaging and left ventricular global longitudinal strain
}

\begin{abstract}
Mohammed El Mahdiui, Jeff M. Smit, Alexander R. van Rosendael, Victoria Delgado, Nina Ajmone Marsan,
\end{abstract} J. Wouter Jukema, Arthur J. H. A. Scholte and Jeroen J. Bax

* Correspondence: j.j.bax@lumc.nl Department of Cardiology, Heart Lung Centre, Leiden University Medical Centre, Albinusdreef 2, 2300 RC Leiden, The Netherlands

\begin{abstract}
Background: Even though implantable cardioverter defibrillator (ICD) implantation for primary prevention has shown to reduce the risk of sudden cardiac death in chronic heart failure patients with reduced left ventricular ejection fraction (LVEF), a significant portion of these patients will never receive appropriate ICD therapy. We aimed to functionally characterize the arrhythmogenic substrate using left ventricular (LV) global longitudinal strain (GLS) and heart-to-mediastinum (H/M) ratio on ${ }^{123}$-metaiodobenzylguanidine $\left({ }^{123}\right.$ I-MIBG) scintigraphy.
\end{abstract}

Methods: We included patients with heart failure with reduced LVEF who received an ICD for primary prevention. To functionally characterize the arrhythmogenic substrate, we measured the LV GLS with two-dimensional speckle tracking echocardiography and cardiac innervation measured as the H/M ratio on ${ }^{123}$ I-MIBG scintigraphy. An event was defined as appropriate ICD therapy.

Results: A total of 155 patients were included, $74 \%$ were male and the mean age was $72 \pm 9$ years. During a median follow-up of 10 (6-12) years, 43 patients (28\%)

experienced appropriate ICD therapy. Patients that experienced an event were more often male, had more often ischaemic cardiomyopathy and were more likely to have worse renal function. There was no difference in the left ventricular ejection fraction (LVEF) between the two groups ( $25 \pm 6.4 \%$ vs $26 \pm 6.0 \%, p=0.276$ ). However, LV GLS was significantly more impaired in the group that experienced an event compared to patients that did not $(-6.7 \pm 2.1 \%$ vs $-7.6 \pm 2.1 \% ; p=0.020)$. The innervation, measured as the $\mathrm{H} / \mathrm{M}$ ratio on ${ }^{123} \mathrm{I}-\mathrm{MIBG}$ scintigraphy was also significantly more impaired in the patients that experienced and event compared to patients that did not (1.34 $\pm 0.2 \mathrm{vs}$ $1.47 \pm 0.2, p \leq 0.001$ ). Multivariable Cox regression analysis showed LV GLS and H/M ratio independently associated with appropriate ICD therapy with a hazard ratio of $1.24(95 \% \mathrm{Cl} 1.027-1.491, p=0.025)$ and $5.71(95 \% \mathrm{Cl} 1.135-28.571, p=0.034)$, respectively. LV GLS and H/M ratio were significantly correlated (Pearson correlation coefficient $-0.30, p<0.001$ ).

Conclusions: Functionally characterizing the arrhythmogenic substrate using different imaging techniques defines the risk for appropriate ICD therapy, whereas LVEF did not.

Keywords: Global longitudinal strain, ${ }^{123}$-meta-iodobenzylguanidine, Sudden cardiac death, Arrhythmogenic substrate 


\section{Background}

Evaluation of heart failure patients who may benefit from implantable cardioverter defibrillator (ICD) remains challenging. Current guidelines recommend an ICD implantation as primary prevention in symptomatic heart failure patients with a left ventricular ejection fraction (LVEF) $\leq 35 \%$ treated with optimal medical therapy (Priori et al. 2015). However, around $40 \%$ of these patients receiving an ICD will not develop ventricular arrhythmias that can be appropriately treated with the ICD (Moss et al. 2004). Therefore, a more individualized risk stratification strategy is necessary.

Characterization of the anatomical and functional substrate that may lead to re-entrant ventricular tachycardia and sudden cardiac death has been shown feasible with various imaging techniques (Bertini et al. 2012). Anatomically, the arrhythmogenic substrate is characterized by bundles of scar, fibrous tissue intermingled with viable myocardium (Fernandez-Armenta et al. 2013; Lin et al. 2013). Functionally, the arrhythmogenic substrate is characterized by heterogeneous regional contraction and denervated myocardium (Boogers et al. 2010; Ersboll et al. 2013; Jacobson et al. 2010). Transient factors, such as ischaemia, can influence this arrhythmogenic substrate and serve as trigger for reentry tachyarrhythmias. Left ventricular (LV) global longitudinal strain (GLS) measured with two-dimensional (2D) speckle tracking echocardiography describes better than the LVEF heterogeneous contraction of the LV myocardium and has been associated with LV myocardial scar burden (Bello et al. 2005; Gulati et al. 2013; Schmidt et al. 2007). In addition, in ischaemic heart failure, the value of longitudinal strain of the peri-infarct zone has been associated with appropriate ICD therapy (Ng et al. 2010). Sympathetic myocardial innervation can be assessed with ${ }^{123}$ I-meta-iodobenzylguanidine $\left({ }^{123} \mathrm{I}\right.$-MIBG) scintigraphy. This radiotracer is an analogue of norepinephrine up taken by the sympathetic nerve terminals of the heart, without being metabolized. In heart failure patients, the uptake of ${ }^{123}$ I-MIBG by the heart is reduced, indicating myocardial denervation. A reduced heart-to-mediastinum $(\mathrm{H} / \mathrm{M})$ ratio of the ${ }^{123} \mathrm{I}$-MIBG uptake on planar imaging has been associated with increased risk of ventricular arrhythmic events (Jacobson et al. 2010; Nagahara et al. 2008). So far, there is no study using multimodality imaging to functionally assess the arrhythmogenic substrate.

The aim of the present study was to functionally characterize the arrhythmogenic substrate of heart failure patients receiving an ICD by measuring LV GLS and the H/M ratio on planar ${ }^{123} \mathrm{I}-\mathrm{MIBG}$ scintigraphy imaging and study the differences between patients presenting with appropriate ICD therapies and patients without.

\section{Methods}

\section{Patients and clinical evaluation}

Heart failure patients who received an ICD for primary prevention, according to prevailing guidelines (Priori et al. 2015), and who underwent clinically indicated a ${ }^{123}$ I-MIBG scintigraphy between 2004 and 2017 at the Leiden University Medical Centre (The Netherlands) were retrospectively evaluated. Demographic and clinical characteristics were collected using the departmental cardiology information system (EPD-vision; Leiden University Medical Centre, Leiden, The Netherlands) and electronic medical records (HiX; ChipSoft, Amsterdam, The Netherlands) and retrospectively analysed. Echocardiographic data were stored in the echocardiographic database and the planar ${ }^{123}$ I-MIBG 
scintigraphy data in the department of nuclear medicine database. Clinical, echocardiographic and ${ }^{123}$ I-MIBG scintigraphy data were analysed retrospectively. The association between clinical, conventional and advanced (2D speckle tracking imaging) echocardiographic variables and ${ }^{123}$ I-MIBG variables and ventricular arrhythmic events was investigated. For retrospective analysis of clinically acquired data anonymously handled, the institutional review board waived the need for written patient informed consent.

\section{Transthoracic echocardiography: conventional and speckle tracking analysis}

Transthoracic echocardiography was performed with the patients at rest, in the left lateral decubitus position using a commercially available system (Vivid 7 and E9, GE Healthcare, Horten, Norway). Bimodal, M-mode, colour, continuous and pulsed wave Doppler data were acquired with $3.5 \mathrm{MHz}$ or M5S transducers and digitally stored in cine-loop format. Offline analysis was performed using the EchoPac system (version BT13, GE Medical Systems, Horten, Norway). Left ventricular end-diastolic volume (LVEDV) and left ventricular end-systolic volume (LVESV) were measured on the apical two- and four-chamber views according to the Simpson biplane method and the LVEF was calculated (Lang et al. 2015).

Using 2D speckle tracking echocardiography, the LV GLS was measured. After manually tracing the endocardial border of the left ventricle in the long-axis, two- and four-chamber views, the regions of interest (ROI) were automatically created and adjusted to the thickness of the myocardium. The software automatically tracks the myocardium throughout the cardiac cycle and the quality of the tracking is evaluated. The LV GLS was calculated as the average of the peak systolic longitudinal strain of the three apical views and the results were displayed in a 17-segment "bull's-eye" plot.

\section{${ }^{123}$ I-MIBG scintigraphy data acquisition and analysis}

For assessment of myocardial innervation, $185 \mathrm{MBq}$ of ${ }^{123} \mathrm{I}$-MIBG (AdreView, GE Healthcare, Princeton, NJ, USA) was injected intravenously, after thyroid blockage with sodium iodide. Using a dual-head gamma camera (GCA-7200, Toshiba Corp., Tokyo, Japan) equipped with a low-energy high-resolution collimators, planar anterior images of the thorax were obtained $4 \mathrm{~h}$ (late) after tracer injection. Images were obtained with a $15 \%$ energy window centred at the $159 \mathrm{keV}$ energy peak of $123 \mathrm{I}$ and were subsequently stored in a $256 \times 256$ matrix.

A ROI was manually drawn over the entire heart to measure the counts in the LV myocardial region. To measure the counts in the mediastinal region, a rectangular ROI was placed on the upper half of the mediastinum using the lung apex, upper cardiac border and medial contours of the lungs as borders. The late $\mathrm{H} / \mathrm{M}$ ratio was calculated by dividing the mean counts in the cardiac ROI by the mean counts in the mediastinal ROI. The analysis was performed using dedicated post-processing software on a Syngo-MI workstation (Siemens Medical Solutions, Malvern, PA, USA).

\section{Follow-up}

Patients were followed up at the heart failure outpatient clinic and the ICD devices were interrogated on 6-monthly visits. The ICD ventricular tachyarrhythmia detection criteria and therapy were programmed conventionally. The occurrence of 
appropriate ICD therapy was the primary endpoint. Appropriate ICD therapy was defined as anti-tachycardia pacing or shock for ventricular tachycardia or ventricular fibrillation.

\section{Statistical analysis}

Continuous variables are reported as mean \pm standard deviation if normally distributed, and as median and $25-75 \%$ interquartile range (IQR) if non-normally distributed. Categorical data are presented as frequencies and percentages. Patients were divided in two groups according to the occurrence of an event, appropriate ICD therapy or not. Continuous data were compared between patients presenting with event and patients without event using the Student's $t$ test or the MannWhitney $U$ test. Categorical data were compared with the $\chi 2$ test. Cox proportional hazard regression analyses were used to evaluate the variables that were significantly associated with appropriate ICD therapy. The relationship between LV GLS and $\mathrm{H} / \mathrm{M}$ ratio was investigated using the Pearson correlation. A $p$ value $<0.05$ was considered statistically significant. All statistical analyses were performed using the SPSS software package (IBM Corp. Released 2015, IBM SPSS Statistics for Windows, Version 23.0. Armonk, NY, USA: IBM Corp.).

\section{Results}

A total of 155 patients with ${ }^{123}$ I-MIBG scintigraphy and echocardiography with LV GLS data were included ( $74 \%$ were male and the mean age was $72 \pm 9$ years). During a median follow-up of 10 years (25-75\% IQR 6-12 years), appropriate ICD therapy occurred in 43 patients (28\%). An ICD shock was the first appropriate ICD therapy in $70 \%$ of the cases. Table 1 shows the baseline clinical characteristics for the total population and the patients who did or did not experience an appropriate ICD therapy. The majority of patients referred had New York Heart Association (NYHA) functional class II or III heart failure symptoms. The two groups of patients were comparable in various demographic and clinical characteristics.

The imaging characteristics are shown in Table 2. Patients with an event had significantly larger LVEDV as compared to patients without an event. However, the groups were comparable in terms of $\operatorname{LVEF}(25 \pm 6.4 \%$ vs $26 \pm 6.0 \% ; p=0.276)$. In contrast, patients who presented with an event showed significantly more impaired LV GLS compared to their counterparts $(-6.7 \pm 2.1 \%$ vs $-7.6 \pm 2.1 \% ; p=0.020$, Fig. 1$)$. In the planar ${ }^{123} \mathrm{I}-\mathrm{MIBG}$ data, the late $\mathrm{H} / \mathrm{M}$ ratio was significantly lower in the group of patients who experienced an event compared to the group who did not $(1.34 \pm 0.2$ vs $1.47 \pm 0.2, p \leq 0.001$, Fig. 1). Multivariate Cox regression analysis showed that LV GLS and $\mathrm{H} / \mathrm{M}$ ratio were independently associated with appropriate ICD therapy, with a hazard ratio of 1.24 (95\% CI 1.027-1.491, $p=0.025$ ) for LV GLS and 5.71 (95\% CI 1.135-28.571, $p=0.034$ ) for H/M ratio when corrected for LVEDV and LVEF.

There was a moderate but significant correlation between LV GLS and H/M ratio $(r=0.30, p<0.001$, Fig. 2). This correlation remained significant in the group that did not experience an event $(r=-0.21, p=0.027)$ and was stronger in the group that did experience an event $(r=-0.50, p=0.002)$ (Fig. 2). 
Table 1 Patient clinical characteristics

\begin{tabular}{|c|c|c|c|c|}
\hline & $\begin{array}{l}\text { All Patients } \\
(n=155)\end{array}$ & $\begin{array}{l}\text { Patients without appropriate } \\
\text { ICD therapy }(n=112)\end{array}$ & $\begin{array}{l}\text { Patients with appropriate } \\
\text { ICD therapy }(n=43)\end{array}$ & $p$-value \\
\hline \multicolumn{5}{|l|}{ Clinical characteristics } \\
\hline Age & $72 \pm 8.9$ & $72 \pm 8.7$ & $71 \pm 9.3$ & 0.700 \\
\hline Male, n(\%) & $115(74)$ & $79(71)$ & $36(84)$ & 0.093 \\
\hline \multicolumn{5}{|l|}{ NYHA functional class, n(\%) } \\
\hline I & $21(14)$ & $16(14)$ & $5(12)$ & \multirow[t]{3}{*}{0.817} \\
\hline$\|$ & $51(33)$ & $35(31)$ & $16(37)$ & \\
\hline III/IV & $80(52)$ & $59(53)$ & $21(49)$ & \\
\hline $\begin{array}{l}\text { Ischemic cardiomyopathy, } \\
n(\%)\end{array}$ & $88(57)$ & $60(54)$ & $28(65)$ & 0.194 \\
\hline \multicolumn{5}{|l|}{ Devices } \\
\hline$I C D, n(\%)$ & $51(33)$ & $36(32)$ & $15(35)$ & \multirow[t]{2}{*}{0.745} \\
\hline CRT-D, n(\%) & $104(67)$ & $76(68)$ & $28(65)$ & \\
\hline \multicolumn{5}{|l|}{ Cardiovascular risk factors } \\
\hline Dyslipidemia, n(\%) & $44(28)$ & $30(27)$ & $14(33)$ & 0.475 \\
\hline Diabetes, n(\%) & $25(16)$ & $19(17)$ & $6(14)$ & 0.648 \\
\hline Hypertension, n(\%) & $57(37)$ & $41(37)$ & $16(37)$ & 0.945 \\
\hline Smoking, n(\%) & $35(23)$ & $25(22)$ & $10(23)$ & 0.901 \\
\hline \multicolumn{5}{|l|}{ Medication } \\
\hline ACE-I/ARB's, n(\%) & $132(85)$ & $97(87)$ & $35(81)$ & 0.414 \\
\hline Diuretics, n(\%) & $115(74)$ & $79(71)$ & $36(84)$ & 0.093 \\
\hline Beta-blockers, n(\%) & $118(76)$ & $83(74)$ & $35(81)$ & 0.341 \\
\hline -Sotalol & $5(4)$ & $4(4)$ & $1(2)$ & 1.000 \\
\hline Calcium-blockers, n(\%) & $77(50)$ & $55(49)$ & $22(51)$ & 0.819 \\
\hline Amiodarone, n(\%) & $30(19)$ & $23(21)$ & $7(16)$ & 0.548 \\
\hline Digoxin, n(\%) & $17(11)$ & $14(13)$ & $3(7)$ & 0.325 \\
\hline Statin, n(\%) & $97(63)$ & $70(63)$ & $27(63)$ & 0.973 \\
\hline Anti-diabetic medication, n(\%) & $83(54)$ & $58(52)$ & $25(58)$ & 0.478 \\
\hline \multicolumn{5}{|l|}{ Laboratory results } \\
\hline Creatinine (mmol/L) & $101 \pm 32$ & $100 \pm 31$ & $105 \pm 35$ & 0.368 \\
\hline Hematocrit (\%) & $41.6 \pm 3.3$ & $41.6 \pm 3.3$ & $41.5 \pm 3.5$ & 0.887 \\
\hline
\end{tabular}

Values are mean \pm standard deviation or $\mathrm{n}(\%)$.

$A C E-I$ Angiotensin converting enzyme inhibitor, $A R B^{\prime}$ 's Angiotensin II receptor blockers, $C R T-D$ Cardiac resynchronization therapy defibrillator, ICD Implantable cardioverter defibrillator, NYHA The New York Heart Association.

\section{Discussion}

Despite showing similar LVEF, heart failure patients experiencing appropriate ICD therapy showed more impaired LV systolic function when measured with 2D speckle tracking GLS and more impaired myocardial innervation as assessed with ${ }^{123}$ I-MIBG scintigraphy. These results provide additional evidence on the limited value of LVEF to identify heart failure patients who will benefit from an ICD.

\section{Assessment of arrhythmogenic substrate}

The structural LV changes that underlie heart failure with reduced LVEF include expansion of extracellular matrix with increase of collagen and fibroblasts. Scar or fibrotic 
Table 2 LV GLS and ${ }^{123}$ I-MIBG characteristics

\begin{tabular}{|c|c|c|c|c|}
\hline & $\begin{array}{l}\text { All Patients } \\
(n=155)\end{array}$ & $\begin{array}{l}\text { Patients without appropriate } \\
\text { ICD therapy }(n=112)\end{array}$ & $\begin{array}{l}\text { Patients with appropriate } \\
\text { ICD therapy }(n=43)\end{array}$ & $p$-value \\
\hline \multicolumn{5}{|c|}{ Two-dimensional echocardiography } \\
\hline $\begin{array}{l}\text { Left ventricular end-diastolic } \\
\text { volume, (ml) }\end{array}$ & $247 \pm 87$ & $236 \pm 83$ & $274 \pm 90$ & 0.017 \\
\hline $\begin{array}{l}\text { Left ventricular end- systolic } \\
\text { volume, (ml) }\end{array}$ & $186 \pm 72$ & $181 \pm 71$ & $201 \pm 73$ & 0.117 \\
\hline $\begin{array}{l}\text { Left ventricular ejection } \\
\text { fraction, (\%) }\end{array}$ & $25 \pm 6.3$ & $25 \pm 6.4$ & $26 \pm 6.0$ & 0.276 \\
\hline Left ventricular GLS, (\%) & $-7.4 \pm 2.2$ & $-7.6 \pm 2.1$ & $-6.7 \pm 2.2$ & 0.020 \\
\hline \multicolumn{5}{|l|}{${ }^{123}$ I-MIBG SPECT } \\
\hline Heart mediastinum ratio late & $1.43 \pm 0.2$ & $1.47 \pm 0.2$ & $1.34 \pm 0.2$ & $<0.001$ \\
\hline
\end{tabular}

Values are mean \pm standard deviation

GLS Global longitudinal strain

tissues (bundles of collagen) are not per se pro-arrhythmic since they are electrically inert tissue. However, the areas where the fibrous/scar tissue intermingles with viable myocytes create a substrate where re-entry circuits may form and lead to ventricular arrhythmias (Morita et al. 2014). After myocardial infarction, this area is known as border zone.

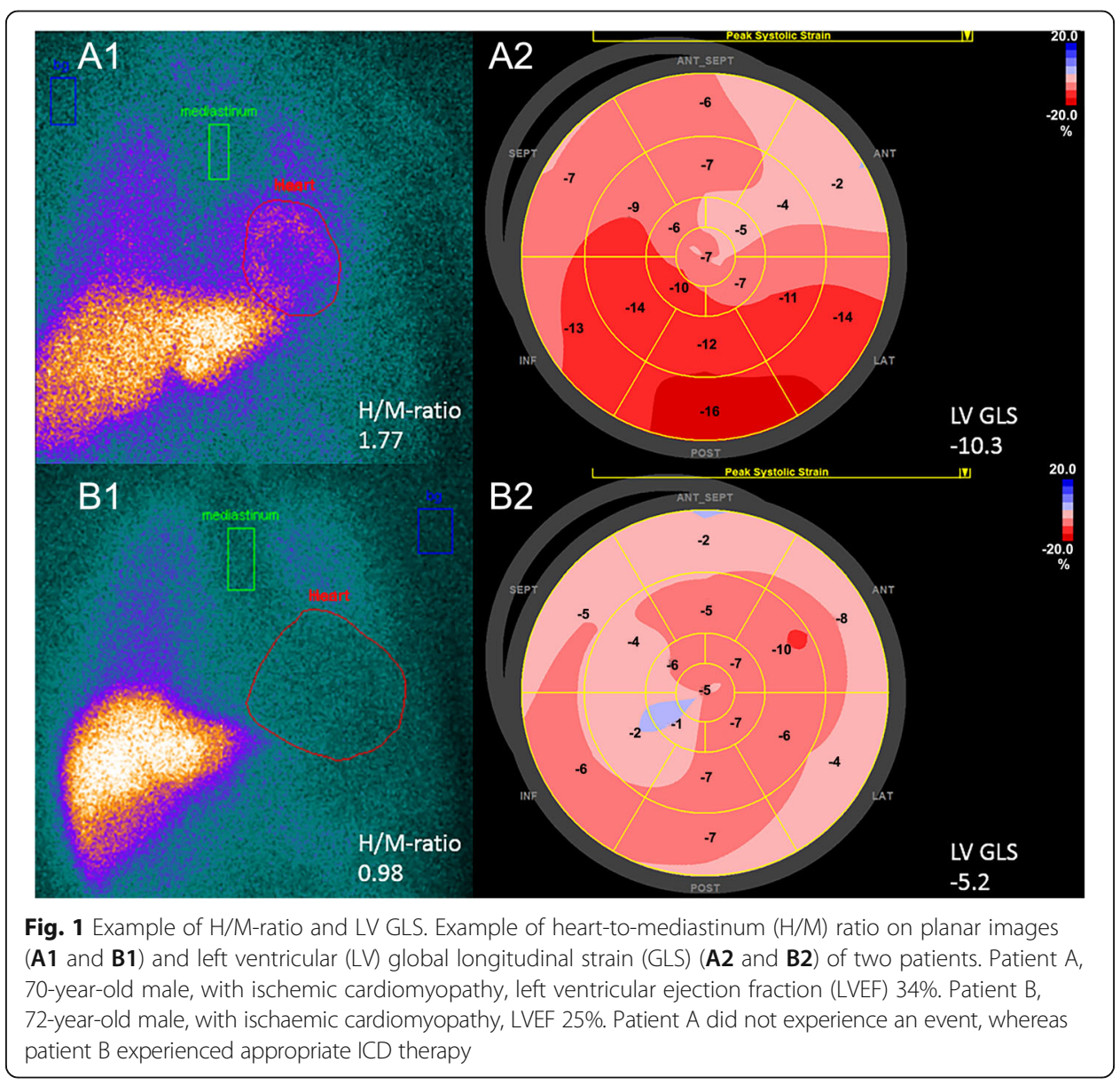



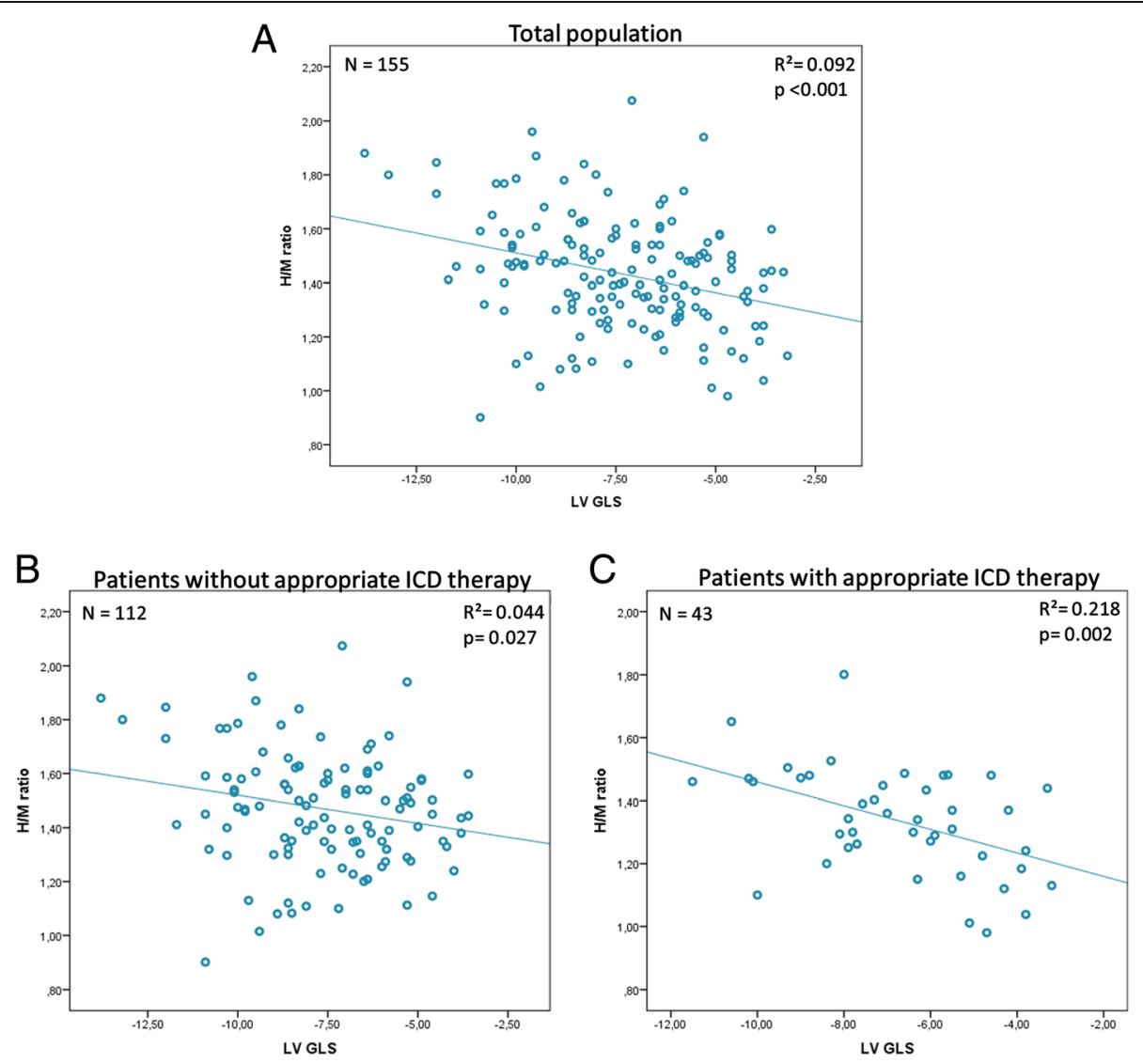

Fig. 2 Correlation between H/M ratio and LV GLS. Correlation between heart-to-mediastinum (H/M) ratio and left ventricular global longitudinal strain (LV GLS) in the total population (a), patients who did not experience an event (b) and patients who did experience an event (c). There was a significant correlation between $\mathrm{H} / \mathrm{M}$ ratio and LV GLS using the Pearson correlation coefficient for the total population $(r=0.30, p<0.001)$, patients who did not experience an event $(r=-0.21, p=0.027)$ and those who did $(r=-0.50, p=0.002)$

The border zone can be characterized using different imaging modalities. On late gadolinium contrast enhanced cardiovascular magnetic resonance (LGE CMR), the border zone shows intermediate signal intensity between normal myocardium and dense myocardial scar. Applying specific thresholds, the amount of border zone can be quantified. In 91 patients with ischaemic heart disease and indication for ICD implantation as primary prevention, Roes et al. demonstrated that each 10-g increase in the mass of border zone was associated with 51\% increased risk of ventricular arrhythmias and appropriate ICD shocks (Roes et al. 2009a). The functional properties of the scar zone, border zone and normal myocardium can be evaluated with 2-dimensional speckle tracking echocardiography and the measurement of LV regional longitudinal strain. In ischaemic heart failure, LV segments with values of longitudinal strain more impaired than $-4.5 \%$ (less negative) have been shown to correspond to areas of transmural scar (sensitivity 81.2\% and specificity 81.6\%) (Roes et al. 2009b). The areas surrounding the LV segments with a value of longitudinal strain more negative than $-4.5 \%$ can be considered border zone. In 424 ischaemic heart failure patients who received an ICD as primary prevention, $\mathrm{Ng}$ et al. showed that the occurrence of ventricular arrhythmias and appropriate ICD shocks during follow-up was more frequent as the longitudinal strain of 
the border zone was more impaired (hazard ratio 1.25) (Ng et al. 2010). The present study provides additional evidence on the association between impaired LV GLS and the occurrence of appropriate ICD therapies. More impaired LV GLS reflects a larger burden of myocardial fibrosis which may lead to areas of slow conduction and conduction block and favour the formation of re-entry circuits and ventricular arrhythmias. In addition, the association between LV GLS and sudden cardiac death, ventricular arrhythmias or appropriate ICD therapy has been also demonstrated in previous studies including 988 patients with acute myocardial infarction and LVEF > 35\% in 91\% of them (Ersboll et al. 2013). On multivariate analysis, LV GLS was independently associated with the occurrence of sudden cardiac death or ventricular arrhythmias with a hazard ratio of 1.24 , similar to the hazard ratio reported in the current study.

Moreover, the border zone is also characterized by viable myocardium with abnormal innervation. Previous studies have shown that in ischaemic cardiomyopathy patients, the areas of denervation exceed the area of perfusion defects on single photon emission tomography (SPECT) (Bax et al. 2008; Gimelli et al. 2014; Matsunari et al. 2000). The higher susceptibility of sympathetic nerve fibres to ischaemia compared to myocytes leads to larger areas of denervation than scar after myocardial infarction (denervation-perfusion mismatch) (Matsunari et al. 2000; McGhie et al. 1991; Zipes 1990). By assessing myocardial denervation with ${ }^{123}$ I-MIBG SPECT and myocardial perfusion with ${ }^{99 \mathrm{~m}}$ Technetiumtetrofosmin SPECT, the mismatch can be quantified. Patients with larger areas of denervation-perfusion mismatch have shown a higher incidence of ventricular arrhythmia events (Boogers et al. 2010). Using planar ${ }^{123}$ I-MIBG scintigraphy, the AdreView Myocardial Imaging for Risk Evaluation in Heart Failure (ADMIRE-HF) study, the largest study so far including 964 heart failure patients followed for 2 years, showed that patients with an $\mathrm{H} / \mathrm{M}$ ratio between 1.2 and 1.6 had more frequent ventricular arrhythmic events as compared to patients with an H/M ratio $\geq 1.6$ (Jacobson et al. 2010). This parameter reflects the global cardiac sympathetic innervation rather than characterizing the border zone where the re-entrant ventricular arrhythmias may start. In the present study, patients who received appropriate ICD therapy showed more impaired $\mathrm{H} / \mathrm{M}$ ratio compared to those who remain without event.

The correlation between LV GLS and H/M ratio was moderate, indicating the distinct differences between both modalities. Our findings are in agreement with previously published studies, reporting similar correlations between LV GLS and H/M ratio (Bulten et al. 2015; Cruz et al. 2019). In clinical practice, a combination of multimodality imaging to characterize the arrhythmogenic substrate from the anatomical and functional point of view may be important to accurately identify the patients who will benefit from an ICD implantation. The present study provides further evidence on the importance of such characterization by combining speckle tracking echocardiography and innervation imaging.

\section{Study limitations}

There are several limitations that should be acknowledged. First, this was a retrospective, single-centre study. Furthermore, since 2D speckle tracking echocardiography requires good image quality for reliable analysis, selection bias might have been introduced. The patient population was heterogeneous, with ischaemic and non-ischaemic 
cardiomyopathy patients. Also, ischaemia might have risen during the long follow-up of the study and have influenced the results. Finally, the event rate for appropriate ICD therapy in our population was relatively low compared to previous study (Moss et al. 2004). This might be reflected by the difference in study populations.

\section{Conclusions}

In conclusion, LV GLS measured by 2D speckle tracking echocardiography and H/M ratio measured by planar ${ }^{123}$ I-MIBG SPECT were significantly more impaired in patients who experienced an appropriate ICD therapy compared to patients that did not. In contrast, no differences were observed between groups of patients in terms of LVEF. Multivariate Cox regression analysis showed LV GLS and H/M ratio to be independent predictors of appropriate ICD therapy. These results underscore the importance of multimodality imaging for the characterization of the arrhythmogenic substrate and the identification of patients who will benefit from an ICD.

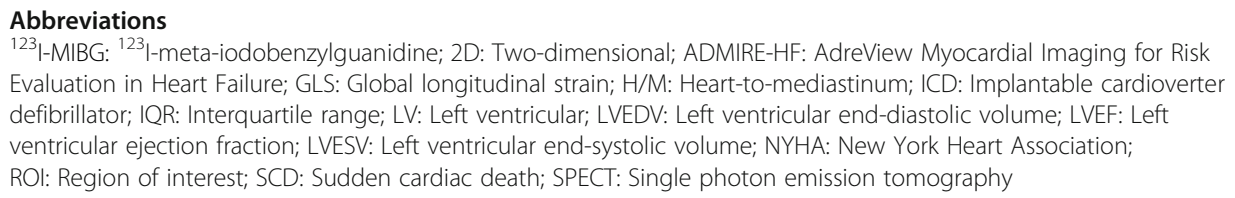

\section{Authors' contributions}

MEM contributed to the conception and design of the manuscript; collection, analysis and interpretation of literature and clinical data; drafting of the manuscript; and final approval of the manuscript. JMS contributed to the conception and design of the manuscript, interpretation of the data, revision of the manuscript and final approval of the manuscript. $A R V R, V D$ and JJB contributed to the conception and design of the manuscript, interpretation of data, revision of the manuscript and final approval of the manuscript. NAM contributed to the conception and design of the manuscript, revision of the manuscript and final approval of the manuscript. JWJ and AJHAS contributed to the revision of the manuscript and final approval of the manuscript. All authors read and approved the final manuscript.

\section{Funding}

There were no sources of funding.

\section{Availability of data and materials}

Data are available from the corresponding authors on a reasonable request.

\section{Ethics approval and consent to participate}

All procedures performed in studies involving human participants were in accordance with the ethical standards of the institutional and/or national research committee and with the 1964 Helsinki Declaration and its later amendments or comparable ethical standards.

\section{Consent for publication}

For retrospective analysis of clinically acquired data anonymously handled, the institutional review board waived the need for written patient informed consent.

\section{Competing interests}

The Department of Cardiology of the Leiden University Medical Centre received research grants from Biotronik, Medtronic, Boston Scientific and Edwards Lifesciences. Victoria Delgado and Nina Ajmone Marsan received speaker fees from Abbott Vascular. Jeroen J Bax received speaker fees from Abbott Vascular and Boehringer Ingelheim. Arthur Scholte received speaker fees from Canon Medical Systems and GE Healthcare. The other authors declare that they have no competing interests.

Received: 22 April 2019 Accepted: 24 June 2019

Published online: 02 August 2019

\section{References}

Bax JJ, Kraft O, Buxton AE, Fjeld JG, Parizek P, Agostini D et al (2008) 123 I-mIBG scintigraphy to predict inducibility of ventricular arrhythmias on cardiac electrophysiology testing: a prospective multicenter pilot study. Circ Cardiovasc Imaging 1:131-140

Bello D, Fieno DS, Kim RJ, Pereles FS, Passman R, Song G et al (2005) Infarct morphology identifies patients with substrate for sustained ventricular tachycardia. J Am Coll Cardiol 45:1104-1108

Bertini M, Schalij MJ, Bax JJ, Delgado V (2012) Emerging role of multimodality imaging to evaluate patients at risk for sudden cardiac death. Circ Cardiovasc Imaging 5:525-535 
Boogers MJ, Borleffs CJ, Henneman MM, van Bommel RJ, van Ramshorst J, Boersma E et al (2010) Cardiac sympathetic denervation assessed with 123-iodine metaiodobenzylguanidine imaging predicts ventricular arrhythmias in implantable cardioverter-defibrillator patients. J Am Coll Cardiol 55:2769-2777

Bulten BF, Verberne HJ, Bellersen L, Oyen WJ, Sabate-Llobera A, Mavinkurve-Groothuis AM et al (2015) Relationship of promising methods in the detection of anthracycline-induced cardiotoxicity in breast cancer patients. Cancer Chemother Pharmacol 76:957-967

Cruz MC, Abreu A, Portugal G, Santa-Clara H, Cunha PS, Oliveira MM et al (2019) Relationship of left ventricular global longitudinal strain with cardiac autonomic denervation as assessed by (123)I-mIBG scintigraphy in patients with heart failure with reduced ejection fraction submitted to cardiac resynchronization therapy : assessment of cardiac autonomic denervation by GLS in patients with heart failure with reduced ejection fraction submitted to CRT. J Nucl Cardiol 26:869-879

Ersboll M, Valeur N, Andersen MJ, Mogensen UM, Vinther M, Svendsen JH et al (2013) Early echocardiographic deformation analysis for the prediction of sudden cardiac death and life-threatening arrhythmias after myocardial infarction. JACC Cardiovasc Imaging 6:851-860

Fernandez-Armenta J, Berruezo A, Andreu D, Camara O, Silva E, Serra L et al (2013) Three-dimensional architecture of scar and conducting channels based on high resolution ce-CMR: insights for ventricular tachycardia ablation. Circ Arrhythm Electrophysiol 6:528-537

Gimelli A, Masci PG, Liga R, Grigoratos C, Pasanisi EM, Lombardi M et al (2014) Regional heterogeneity in cardiac sympathetic innervation in acute myocardial infarction: relationship with myocardial oedema on magnetic resonance. Eur J Nucl Med Mol Imaging 41:1692-1694

Gulati A, Jabbour A, Ismail TF, Guha K, Khwaja J, Raza S et al (2013) Association of fibrosis with mortality and sudden cardiac death in patients with nonischemic dilated cardiomyopathy. JAMA 309:896-908

Jacobson AF, Senior R, Cerqueira MD, Wong ND, Thomas GS, Lopez VA et al (2010) Myocardial iodine-123 metaiodobenzylguanidine imaging and cardiac events in heart failure. Results of the prospective ADMIRE-HF (AdreView Myocardial Imaging for Risk Evaluation in Heart Failure) study. J Am Coll Cardiol 55:2212-2221

Lang RM, Badano LP, Mor-Avi V, Afilalo J, Armstrong A, Ernande L et al (2015) Recommendations for cardiac chamber quantification by echocardiography in adults: an update from the American Society of Echocardiography and the European Association of Cardiovascular Imaging. J Am Soc Echocardiogr 28:1-39 e14

Lin LY, Su MY, Chen JJ, Lai LP, Hwang JJ, Tseng CD et al (2013) Conductive channels identified with contrast-enhanced MR imaging predict ventricular tachycardia in systolic heart failure. JACC Cardiovasc Imaging 6:1152-1159

Matsunari I, Schricke U, Bengel FM, Haase HU, Barthel P, Schmidt G et al (2000) Extent of cardiac sympathetic neuronal damage is determined by the area of ischemia in patients with acute coronary syndromes. Circulation 101:2579-2585

McGhie Al, Corbett JR, Akers MS, Kulkarni P, Sills MN, Kremers M et al (1991) Regional cardiac adrenergic function using I-123 meta-iodobenzylguanidine tomographic imaging after acute myocardial infarction. Am J Cardiol 67:236-242

Morita N, Mandel WJ, Kobayashi Y, Karagueuzian HS (2014) Cardiac fibrosis as a determinant of ventricular tachyarrhythmias. J Arrhythm 30:389-394

Moss AJ, Greenberg H, Case RB, Zareba W, Hall WJ, Brown MW et al (2004) Long-term clinical course of patients after termination of ventricular tachyarrhythmia by an implanted defibrillator. Circulation 110:3760-3765

Nagahara D, Nakata T, Hashimoto A, Wakabayashi T, Kyuma M, Noda R et al (2008) Predicting the need for an implantable cardioverter defibrillator using cardiac metaiodobenzylguanidine activity together with plasma natriuretic peptide concentration or left ventricular function. J Nucl Med 49:225-233

Ng AC, Bertini M, Borleffs CJ, Delgado V, Boersma E, Piers SR et al (2010) Predictors of death and occurrence of appropriate implantable defibrillator therapies in patients with ischemic cardiomyopathy. Am J Cardiol 106:1566-1573

Priori SG, Blomstrom-Lundqvist C, Mazzanti A, Blom N, Borggrefe M, Camm J et al (2015) 2015 ESC Guidelines for the management of patients with ventricular arrhythmias and the prevention of sudden cardiac death: the task force for the management of patients with ventricular arrhythmias and the prevention of sudden cardiac death of the European Society of Cardiology (ESC). Endorsed by: Association for European Paediatric and Congenital Cardiology (AEPC). Eur Heart J 36:2793-2867

Roes SD, Borleffs CJ, van der Geest RJ, Westenberg JJ, Marsan NA, Kaandorp TA et al (2009a) Infarct tissue heterogeneity assessed with contrast-enhanced MRI predicts spontaneous ventricular arrhythmia in patients with ischemic cardiomyopathy and implantable cardioverter-defibrillator. Circ Cardiovasc Imaging 2:183-190

Roes SD, Mollema SA, Lamb HJ, van der Wall EE, de Roos A, Bax JJ (2009b) Validation of echocardiographic two-dimensional speckle tracking longitudinal strain imaging for viability assessment in patients with chronic ischemic left ventricular dysfunction and comparison with contrast-enhanced magnetic resonance imaging. Am J Cardiol 104:312-317

Schmidt A, Azevedo CF, Cheng A, Gupta SN, Bluemke DA, Foo TK et al (2007) Infarct tissue heterogeneity by magnetic resonance imaging identifies enhanced cardiac arrhythmia susceptibility in patients with left ventricular dysfunction. Circulation 115:2006-2014

Zipes DP (1990) Influence of myocardial ischemia and infarction on autonomic innervation of heart. Circulation 82:1095-1105

\section{Publisher's Note}

Springer Nature remains neutral with regard to jurisdictional claims in published maps and institutional affiliations. 